\title{
The Reliability and Validity of the Persian Version of Self- Compassion Scale-Revised Edition
}

\author{
Arash Jelodari ${ }^{1 *}$, Samira Gheydari ${ }^{2}$
}

\section{ABSTRACT}

The current study was conducted with the aim of examining the confirmatory factor structure of the revised Persian version of self-compassion rating scale among a group of male prisoners of Sepidar prison of Ahvaz. One hundred and seventy-eight male prisoners were selected using convenient sampling method among male prisoners and completed RPVSC and GHQ-11-item. The confirmatory factor analysis confirmed 6 factors. The Cronbach's $\alpha$ coefficient for the entire scale was .91, for the 6 factors was from .77 to .92 and its convergent validity with the General Health Questionnaire was -.45 and for the 6 subscales with the GHQ was calculated from -.28 to -.48. Finally, the self-compassion scale can be used as a validated instrument in assessing aspects of self-compassion in male prisoners population.

Keywords: Self-Compassion, Prisoners, Reliability, Persian, Validating.

People experience negative emotions such as sadness, burnout, and failure in their lives. In order to cope with these negative emotions, they need to alleviate and eliminate, and above the all, they need to overcome such emotions without any harm. These people need to promote their self-compassion in relation with this type of negative emotions. The term self-compassion is derived from the word "compassion" as it involves being sensitive to others' pain and suffering, being aware of others' discomfort, and the desire to alleviate others' suffering and having a nonjudgmental understanding for the others' mistakes (Deniz, Kesici, \& Sümer, 2008). However, the term has conceptualized by Neff based on Buddhist philosophy. According to Fernando (2003), the ideals of western traditional health system emphasis on self-efficacy, personal independency, productivity, and self-esteem. In contrast, in the ideals of eastern traditional health system the emphasis is on coordination, social integration, balanced performance, protection and care. Salzberg (1997) argues that self-compassion is as that much important in eastern traditions and cultures such as Buddhism. According to this view, the self and the other are interdependent.

\footnotetext{
${ }^{1}$ B.A. student in Counseling Psychology, Shahid Chamran University of Ahvaz

${ }^{2}$ B.A. in General Psychology, PayamNur Tehran Markaz University of Tehran

*Responding Author

(C) 2016 I A Jelodari, S Gheydari; licensee IJIP. This is an Open Access Research distributed under the terms of the Creative Commons Attribution License (http://creativecommons.org/licenses/by/2.0), which permits unrestricted use, distribution, and reproduction in any Medium, provided the original work is properly cited.
} 


\section{The Reliability and Validity of the Persian Version of Self-Compassion Scale-Revised Edition}

Thus, it is not possible to be compassionate about others but not about self. Neff (2003a, b) defines self-compassion as ability to keep one's feelings from suffering along with a sense of fun, communication, and love.

Self-compassion is a relatively new concept in social, personality, and clinical psychology (Leary, Tate, Adams, Batts Allen, \& Hancock, 2007). Neff (2003a, b) argued that selfcompassion has 3 main components which includes: (1) Self-kindness. Since self-criticism and self-judgment originate from feeling of alienation and weak relationships with others (Mongrain, Vettese, Shuster, \& Kendal, 1998), people who are kindly with themselves have not intense judgment and criticism toward themselves (Neff, 2003a; Neff, Hsieh, \& Dejitterat, 2005). The self-criticism of people forms in two related processes. In the first process, self-criticism and enmity create a background which appears under self-hatred. And in the second process, people cannot be warm, soothing and reassuring, self-loving and self-directing. (2) Human community. This humanity community characteristics inspires from culture, human values, justice, independence, and tolerance. People with human community characteristic keep their cultural values, respect to the cultural values of other countries, believe in basic values such as justice, equality, and freedom, and try to adjust their relationship with their values. These people tolerate both themselves and others (Neff, 2001, 2003a, b, c; Neff \& Harter, 2002a, b, 2003; Neff \& Helwig, 2002). And (3) Mindfulness. Mindfulness refers to keeping the painful thoughts and emotions balanced in consciousness instead of over-identification. This process of consciousness reduces the self-criticism, keeps the individual away from negative judgment, and increases the personal perception. When the process occurs, self-kindness increases (Neff, 2003a).

Neff (2003b) developed a scale to measure this personality construct (self-compassion). Since the 2003, much studies have used Self-Compassion Scale as an instrument regarding to varying psychological phenomena and instruments and tests. The principal form of self-compassion includes 26 items which measure 6 components of self-compassion: Self-kindness (When I'm going through a very hard time, I give myself the caring and tenderness I need.), Self-judgment (I don't judge about my own flaws and inadequacies), Human community (I try to see my failings as part of the human condition), Isolation (When I fail at something that's important to me, I tend to feel alone in my failure), Mindfulness (When I fail at something important to me I try to keep things in perspective), and over-identification (When I'm feeling down I tend to obsess and fixate on everything that's wrong). The questions are ranged on a 5-point Likert scale from $1=$ almost never to $5=$ almost always (Neff \& Vonk, 2009). A Dutch version of the scale including 24 items ranging on a 7-point Likert scale has been developed that is very similar to the original version of self-compassion.

The psychometric properties of the scale have been examined in different studies. Neff (2003b) reported the Cronbach's $\alpha$ of .92 for the entire scale, .78 for the subscale of self-kindness, .77 for self-judgment, .80 for human community, .79 for isolation, .75 for mindfulness, and .81 for over- 


\section{The Reliability and Validity of the Persian Version of Self-Compassion Scale-Revised Edition}

identification, and reported test-retest coefficients of .93 for the entire scale and $.88, .88, .80, .85$, .85 , and .88 for the 6 subscales, respectively; Raque-Bogdan, Ericson, Jackson, Martin, and Bryan (2011) reported the Cronbach's $\alpha$ of .92; Raes, Pommier, Neff, and Van Gucht (2011) reported the Cronbach's $\alpha$ of .90 for the long form and from .71 to .83 for its 6 subscales and from .55 to .81 for the 6 subscales in the short form; Deniz, Kesici, and Sumer (2008) reported $.83(\mathrm{p}<.001)$ and .70 for the test-retest reliability coefficient and the Cronbach's $\alpha$ of the scale, respectively; Neff, Kirkpatrick, and Rude (2007) reported the Cronbach's $\alpha$ of .94; In two studies, Wei, Liao, Ku, and Shaffer (2011) reported the Cronbach's $\alpha$ coefficients of .58 and .88 in students and adults populations, respectively.

Using the confirmatory factor analysis method, Neff (2003b), Raes (2010), Raes et al. (2011), Azizi, Mohamad Khani, Foroughi, Lotfi, and Bahram Khani (2013), and Ovec, Akin, and Abaci (2007, cited from Deniz et al., 2008) obtained a 6-factor solution about the Self-Compassion Scale (self-kindness, self-judgment, human community, isolation, mindfulness, and overidentification). But Deniz et al. (2008) did not confirmed 6-factor structure of the SCS. They initially observed a 5 -factor structure with eigenvalues higher than one. But using Scree test, they showed a separate factor and omission of two items out of 26 items which had loadings less than .30 .

Deniz et al. (2008) indicated that the validity coefficients between the SCS and Rosenberg Selfesteem Scale (RSE), Satisfaction with Life Scale (SWLS), and Positive and Negative Affect Schedule (PANAS) were significant. Raes et al. (2011) found a nearly full correlation between the long and short forms of SCS as well as high correlations were observed between two subscales of the forms. Neff (2003b) showed that the SCS has significant, negative correlations with Beck Depression Inventory (BDI), the Speilberger Trait Anxiety (STAI), and Neurotic Perfectionism and has significant, positive correlations with SWLS and Emotional Processing. Van Dam, Sheppard, Forsyth and Earleywine (2011) found that the SCS better can predict people's intense signals and quality of life than the Mindful Attention Awareness Scale (MAAS) does. Ying (2009) indicated that the over-identification subscale is related to depression symptoms. Researchers have shown that self-compassion is related to psychological well-being and can be a protective and nurturing factor of emotional flexibility (Neff \& Vonk, 2009). Also, high levels of self-compassion are related to increased mental health and to decreased anxiety and depression (Leary et al., 2007; Neff, 2003; Neff et al., 2007; Raes, 2010) and to increased happiness and optimistic (Neff et al., 2007).

However, people can keep their psychological well-being and psychological health in family, society, and work settings by having various personality traits including self-compassion. Of course, the assessment of the construct in Iranian society requires a validated and reliable measurement. The current study was developed with two aims of examining the reliability and 
the confirmatory construct of the scale based on the model of Raes et al. in 2011 in the male prisoners population.

\section{METHOD}

\section{Participants}

The statistical population of the study included all of the male and female prisoners (2650 persons, 2450 males and 250 females) of Sepidar prison in Ahvaz in 2014 from which 300 prisoners were chosen convenient and with full consent using the criterion of being married and having at least diploma of which only 178 male persons collaborated. Ninety-four persons (52.8\%) were in range of 20 -30 years old, 47 persons (26.4\%) in 30-40, 20 people $(11.2 \%)$ in 40 50 , 10 people (5.6\%) in 50-60, and 7 people (3.9\%) in 60-70; One hundred and forty-six people (82\%) were diploma, 15 (8.4\%) were associate degree, and 17 (9.6\%) were bachelor; Eighty six people (48.3\%) have been convicting for less than 1 year, 55 (30.9\%) were between 1 or 2 years, and 37 (20.8\%) have been convicting more than 2 years; Fifty persons (28.1\%) were childless, 59 (33.1\%) had 1 child, 35 (19.7\%) had 2 children, 17 (9.6\%) had 3 children, 8 (4.5\%) had 4 children, 4 (3.2\%) had 5 children, 2 (1.6\%) had 6 children, 1 (.6\%) had 8 children, 1 (.6\%) had 12 children, and 1 (.6\%) had 13 children; Thirty-nine (21.9\%) had robbery offenses, 33 (18.5\%) had financial crime, 37 (20.8\%) had drug offenses, 28 (15.7\%) had strife and conflict crimes, 5 (2.8\%) with the crimes of murder, 10 (5.6\%) with an offense of carrying gun, $3(1.7 \%)$ with crime of adultery, 4 (2.2\%) with security-related offenses, 2 (1.1\%) had kidnapping crime, 7 (3.9\%) with forged documents, 8 (4.5\%) had accidents crimes, and 2 (1.1\%) had the dowry crime.

\section{Measures}

Self-Compassion Scale. The 12-item short form of the scale, developed by Raes et al. in 2011, was used to measure the 6 components of self-compassion each of which includes 2 items in the current study. The items are range on a 5-point Likert scale from $1=$ almost never to $5=$ almost always and higher score represents higher levels of self-compassion. However, the scores of selfjudgment, isolation, and over-identification are calculated inversely. The questionnaire was initially translated into Persian and was tested in terms of content and word selection and the Persian text translated into English again by a professor fluent in English and was used with the minimal changes.

General Health Questionnaire-11. The questionnaire has 11 items and each item assess the intense of a psychological problem in last weeks in a 3-rating Likert Scale ( $0=$ less than usual, 1 = not more than usual, 2 = more than usual, and 3 = much more than usual). The minimum and maximum of scores are 0 and 33, respectively, which higher score indicates worse psychological health status (Rajabi \& Hashemi Sheykhshbani, 2009). In a study using exploratory factor analysis on the scale (12 items), with omission of 1 item, 2 factor of social dysfunction (1, 2, 3, 6,7 , and 11) and psychological distress $(4,5,8,9$, and 10) were identified. The Cronbach's $\alpha$ coefficient of the scale was .85, for these two factors was .79 and .84 and the convergent validity coefficient of the scale with the 5-item Mental Health Questionnaire was .73 ( $<<.001)$ and was significant with the mentioned two factors (Rajabi \& Hashemi Sheykhshbani, 2009). 
The Reliability and Validity of the Persian Version of Self-Compassion Scale-Revised Edition

\section{Data analysis}

The data analysis was done using the confirmatory factor analysis for examine and fitness of the 6-fator model of the scale, the Cronbach's $\alpha$ coefficient and Pearson's correlation coefficient for examine convergent the scale with the 11-item Mental Health Questionnaire (Rajabi \& Hashemi Sheykhshbani, 2009). The data were analyzed using Amos and SPSS-21.

\section{RESULTS}

Table 1. The mean, standard deviation, and correlation coefficients between the items and the total score of the SCS

\begin{tabular}{|l|l|l|l|l|l|}
\hline Items & Mean (std. dv.) & Total score & Items & Mean (std. dv.) & Total score \\
\hline 1 & $(1.08) 2.06$ & $.62^{*}$ & 7 & $(1.14) 9.29$ & $.78^{*}$ \\
\hline 2 & $(1.06) 4.40$ & $.61^{*}$ & 8 & $(1.14) 8.28$ & $.75^{*}$ \\
\hline 3 & $(1.14) 2.33$ & $.79^{*}$ & 9 & $(1.03) 6.71$ & $.66^{*}$ \\
\hline 4 & $(1.16) 2.08$ & $.76^{*}$ & 10 & $(1.13) 2.33$ & $.62^{*}$ \\
\hline 5 & $(1.17) 2.33$ & $.64^{*}$ & 11 & $(1.18) 2.10$ & $.72^{*}$ \\
\hline 6 & $(1.15) 8.45$ & $.69^{*}$ & 12 & $(1.25) 2.14$ & $.75^{*}$ \\
\hline Total & $(9.65) 26.69$ & & & \\
\hline
\end{tabular}

$(\mathrm{p}<.01)$

As it is shown in Table 1, the most means are related to item 7 'mindfulness' (9.9) and 6 'selfkindness' (8.45), in order, and the least ones are related to item 1 'over-identification' (2.06) and item 4 'isolation' (2.08), in order. The mean and standard deviation of the entire scale obtained 26.69 and 9.65, respectively. Also, all of the items are significantly related to the score of the entire scale in statistical level of $\mathrm{p}<.01$ (from .61 to .79).

Table 2. The Cronbach's $\alpha$ and convergent validity coefficients of SCS with GHQ-11

\begin{tabular}{|l|l|l|}
\hline Factor & Cronbach's $\boldsymbol{\alpha}$ & Convergent validity \\
\hline Self-compassion & .91 & $-.45^{*}$ \\
\hline Over-identification & .77 & $-.29^{*}$ \\
\hline Self-kindness & .83 & $-.37^{*}$ \\
\hline Mindfulness & .92 & $-.45^{*}$ \\
\hline Isolation & .88 & $-.29^{*}$ \\
\hline Human community & .91 & $-.48^{*}$ \\
\hline Self-judgment & .87 & $-.28^{*}$ \\
\hline
\end{tabular}
( $(\mathrm{p}<.01)$

As it can be seen in table 2, the Cronbach's $\alpha$ coefficients for the entire scale were .91 and for the six factors were .77 for over-identification, .83 for self-kindness, .92 for mindfulness, .88 for

(c) The International Journal of Indian Psychology, ISSN 2348-5396 (e)| ISSN: 2349-3429 (p) | 127 
isolation, .91 for human community, .87 for self-judgment. The convergent validity coefficients of the scale and the six subscales with GHQ-11 (Rajabi \& Hashemi Sheykhshbani, 2009) from $.28(\mathrm{p}<.036)$ to $-.48(\mathrm{p}<.001)$ were significant (the negative signs between the constructs does not implicate that there are negative and inverse relationship, rather it is due to the scoring system of GHQ, namely, the lower score indicates the better mental health).

Table 3. The fitness indices of the 6-factor model of the SCS

\begin{tabular}{|l|l|l|l|l|l|l|l|l|}
\hline$\chi^{2}$ & df & $\mathbf{P}$ & $\chi^{2} /$ d $)$ & GFI & AGFI & CFI & RMR & RMSEA \\
\hline 49.23 & 38 & .105 & 1.29 & .96 & .91 & .99 & .03 & .04 \\
\hline
\end{tabular}

As it is shown in table 3 , the fitness index of $\chi^{2}$ of the 6-factor model is $49.23(\mathrm{p}>.050)$ and is not statistically significant, and indicates that the model is fit to the population. It should be noted that by applying a correction index from $\mathrm{e}_{2}$ to $\mathrm{e}_{10}$, other indices including $\chi^{2} / d f 1.29$ less than the contractual value of 3 , fitness indices of GFI, CFI, and AGFI higher than .90, RMSEA less than .05, RMR are in low which all of them indicate that the 6-factor model with a correction is fit to the population (see figure 1).

\section{(Figure 1. here)}

\section{DISCUSSION}

The current study was done with the aim of examining the validity and reliability of the Persian version of self-compassion scale among a group of prisoners of Sepidar prison of Ahvaz. The key findings can be summarized as follow: First, the results of the structure of confirmatory factor analysis with a correction indicated the fitness of the 6-factor model (factor loads from .78 for the item 1 'over-identification' to .93 for the item 3 'mindfulness'). RMSEA is at the least possible indicting the low error of the model (see table 3). Other fitness indices of the model including GFI $=.96$, AGFI $=.91$, NFI $=.96$, and CFI $=.99$, were all higher than the contractual value of .90 . Also, the value of $\chi^{2}$ is 49.23 and $\chi^{2} / d f$ is less than 3 , which are the other two acceptable fitness indices of the 6-factor model. As a result, this study and the studies of Raes (2010), Raes et al. (2011), Azizi et al. (2013), Neff (2003b), and Ovek et al. (2007, cited from Deniz et al., 2008) have confirmed the structure of the 6-fator model of the Persian version of self-compassion scale, but Deniz et al. (2008) have confirmed the 1-factor model. However, it seems that this personality instrument is applicable in Iranian society even in prisoner participants.

Second, the results of the correlation coefficients for examining the convergent validity showed that there are significant correlations between this scale and its subscales with mental health questionnaire, which the most correlations were related to the subscales of human community and mindfulness. The following studies confirm the finding: Neff (2003b) found a significant, positive relationship between the SCS and Satisfaction with Life Scale and Emotional 


\section{The Reliability and Validity of the Persian Version of Self-Compassion Scale-Revised Edition}

Processing, and a negative relationship with Beck Depression Inventory, the Speilberger Trait Anxiety, and Neurotic Perfectionism; Neff et al. (2007), Leary et al. (2007), Neff (2003), and Raes (2010) found that high levels of self-compassion is related to more mental health and less anxiety and depression and more happiness and optimism; Deniz et al. (2008) found a positive correlation between the SCS and Rosenberg Self-Esteem and Satisfaction with Life scales; Raes et al. (2011) found a correlation between the long and short forms of the SCS as well as the high correlations between two subscales of the long and short forms of SCS. Similarly, Ying (2009) showed that the subscale over-identification is related to depression symptoms. Another study showed that self-compassion is related to psychological well-being and it can be a protective and nurturing factor of emotional flexibility (Neff \& Vonk, 2009).

Third, the Cronbach's $\alpha$ coefficients for the entire SCS and for its 6 subscales were acceptable (see table 2). Given that he Cronbach's $\alpha$ coefficients of .60 and higher are generally acceptable, the finding and other findings of the other studies, such as Deniz et al. (2008), Raes (2010), Raes et al. (2011), Neff (2003), and Azizi et al. (2013), in relation with internal consistency confirm that the reliability levels of the scale are acceptable and the total score and all of the subscales can be used in different contexts of therapy and research.

The 12-item form of SCS can be efficiently used as an alternative instrument instead of the long one to save time and money in therapeutic and research situations and contexts. It can be useful for therapists seeking progression and revision of therapy and counseling activities of patients and clients and helping to them. This version might be a convenient instrument for identifying people developing negative emotions toward themselves and people who cannot develop selfunderstanding and tolerance in Iranian culture. Also, we hope that the revision of the selfcompassion construct can have a contribution in increasing movement of 'positive psychology.' The movement is based on humans' strengths and potentials such as their ability to happiness, love, and forgiveness rather than pathology and maladaptive functioning. However, selfcompassion improves the positive emotions toward self while simultaneously maintains closeness to the others.

However, the generalization of the findings to the other populations should be done with caution, because the chosen sample is not a random one. It is recommended to use other populations in sampling in future studies in order to generalize the findings. We suggest to use retest reliability coefficient to examine the reliability and to use the Rosenberg Self-Esteem Scale to examine the validity of the scale in another study.

\section{Acknowledgments}

We are thankful to the supervisor of Sepidar prison and the prisoners who had collaborated in the research. In addition, the research is done using personal funds and without financial and spiritual resources from any organization. 


\section{REFERENCES}

Andréasson, P. D. (2012). Validation the self-compassion scale: Correlation with the Beck Depression Inventory-II. A Thesis for the Degree Master of Science in Psychology. University, San Luis Obispo.

Azizi, A., Mohammadkhani, P., Foroughi, A. A., Lotfi, S., \& Bahram Khani, M. (2013). The validity and reliability of the Iranian version of the Self -compassion Scale. Iranian Journal of Clinical Psychology, 2, 3, 118-230.

Deniz, M. E., Kesici, Ş., \& Sümer, A. S. (2008). The Validity and Reliability of the Turkish Version of the Self-Compassion Scale. Social Behavior and Personality, 36(9), 11511160.

Fernando, S. (2003). Cultural Diversity, Mental Health and Psychiatry: The struggle against racism. Brunner- Routeledge, New York.

Leary, M. R., Tate, E. B., Adams, C. E., Batts Allen, A., \& Hancock, J. (2007). Self-compassion and reactions to unpleasant self-relevant events: The implications of treating oneself kindly. Journal of Personality and Social Psychology, 92, 887-904.

Mongrain, M., Vettese, L. C., Shuster, B., \& Kendal, N. (1998). Perceptual biases, affect, and behavior in the relationships of dependents and self-critics. Journal of Personality and Social, Psychology, 75, 230-241.

Neff, K. (2003 $)$. Understanding how universal goals of independence and interdependence are manifested within particular cultural contexts. Human Development, 46(5), 312-318.

Neff, K. D. (2003a). Development and validation of a scale to measure self-compassion. Self and Identity, 2, 223-250.

Neff, K. D. (2003a). Self-compassion: An alternative conceptualization of a healthy attitude toward oneself. Self and Identity, 2(2), 85-101.

Neff, K. D. $\left(2003_{\mathrm{b}}\right)$. The development and validation of a scale to measure self-compassion. Self and Identity, 2(3), 223-250.

Neff, K. D. (2004). Self-compassion and psychological well-being. Constructivism in the Human Sciences. 9(2), 27-37.

Neff, K. D., \& Harter, S. (2002 ${ }_{\mathrm{a}}$ ). The authenticity of conflict resolutions among adult couples: Does women's other-oriented behavior reflect their true selves? Sex Roles, 47, 403-417.

Neff, K. D., \& Harter, S. (2002b). The role of power and authenticity in relationship styles emphasizing autonomy, connectedness, or mutuality among adult couples. Journal of Social and Personal Relationships, 19, 835-858.

Neff, K. D., \& Harter, S. (2003). Relationship styles of self-focused autonomy, other-focused connectedness, and mutuality across multiple relationship contexts. Journal of Social and Personal Relationships, 20, 81-99.

Neff, K. D., \& Helwig, C. C. (2002). A constructivist approach to understanding the development of reasoning about rights and authority within cultural contexts. Cognitive Development, 17, 1429-1450. 


\section{The Reliability and Validity of the Persian Version of Self-Compassion Scale-Revised Edition}

Neff, K. D., \& Vonk, R. (2009). Self-compassion versus global self-esteem: Two different ways of relating to oneself. Journal of Personality, 77, 23-50.

Neff, K. D., Hsieh, Y., \& Dejitterat, K. (2005). Self-compassion, achievement goals, and coping with academic failure. Self and Identity, 4(3), 263-287.

Neff, K. D., Kirkpatrick, K. L., \& Rude, S. S. (2007). Self-compassion and adaptive psychological functioning. Journal of Research in Personality, 41, 139-154.

Neff, K. D., Rude, S. S., \& Kirkpatrick, K. L. (2007). An examination of self-compassion in relation to positive psychological functioning and personality traits. Journal of Research in Personality, 41, 908-916.

Ovec, U., Akin, A., \& Abaci, R. (2007). Oz-duyarhk Olceugi: Gecerlik ve Guvenilik Calismasi [Self- Compassion Scale: Validity and reliability study], XVI. Ulusal Egitim Bilmleri Kongresi, Tokat, Turkey. In M. E. Deniz, Ş. Kesici, \& A. S. Sümer. (2008). The Validity and Reliability of the Turkish Version of the Self-Compassion Scale. Social Behavior and Personality, 36(9), 1151-1160.

Raes, F. (2010). Rumination and worry as mediators of the relationship between self-compassion and depression and anxiety. Personality and Individual Differences, 48, 757-761.

Raes, F., Pommier, E., Neff, K. D., \& Van Gucht, D. (2011). Construction and factorial validation of a Short Form of the Self-compassion Scale. Clinical Psychology and Psychotherapy, 18, 250-255.

Rajabi, G., \& Hashemi Sheykhshbani, S. S. (2009). Factor Structure of the 12-item General Health Questionnaire. Journal of Education \& Psychology, 3 (2), 81-94.

Raque-Bogdan, T. L., Ericson, S. K., Jackson, J., Martin, H. M., \& Bryan N. A. (2011). Attachment and mental and physical health: Self-compassion and mattering as mediators. Journal of Counseling Psychology, 58, 2, 272-278.

Salzberg, S. (1997). Loving-kindness: The revolutionary art of happiness. Boston: Shambala.

Shapiro, S. L., Astin, J. A., Bishop, S. R., \& Cordova, M. (2005). Mindfulness-based stress reduction for health care professionals: Results from a randomized trial. International Journal of Stress Management, 12, 164-176.

Van Dam, N., Sheppard, S., Forsyth, J., \& Earleywine, M. (2011). Self-compassion is a better predictor than mindfulness of symptom severity and quality of life in mixed anxiety and depression. Journal of Anxiety Disorders, 25(1), 123-130.

Wei, M., Liao, K. Y-H., Ku, T-Y., \& Shaffer, P. A. (2011).Attachment, self-compassion, empathy, and subjective well-being among college students and community adults. Journal of Personality, 79 (1), 191-221.

Ying, Y. (2009). Contribution of self-compassion to competence and mental health in social work students. Journal of Social Work Education, 45(2), 309.

\section{The items of the Persian version of Self-compassion Scale}

1- When I fail at something important to me I become consumed by feelings of inadequacy.

2- I try to be understanding and patient towards those aspects of my personality I don't like.

(c) The International Journal of Indian Psychology, ISSN 2348-5396 (e)| ISSN: 2349-3429 (p) | 131 


\section{The Reliability and Validity of the Persian Version of Self-Compassion Scale-Revised Edition}

3- When something painful happens I try to take a balanced view of the situation.

4- When I'm feeling down, I tend to feel like most other people are probably happier than I am.

5- I try to see my failings as part of the human condition.

6- When I'm going through a very hard time, I give myself the caring and tenderness I need.

7- When something upsets me I try to keep my emotions in balance.

8- When I fail at something that's important to me, I tend to feel alone in my failure.

9- When I'm feeling down I tend to obsess and fixate on everything that's wrong.

10- When I feel inadequate in some way, I try to remind myself that feelings of inadequacy are shared by most people.

11- I'm disapproving and judgmental about my own flaws and inadequacies.

12- I'm intolerant and impatient towards those aspects of my personality I don't like.

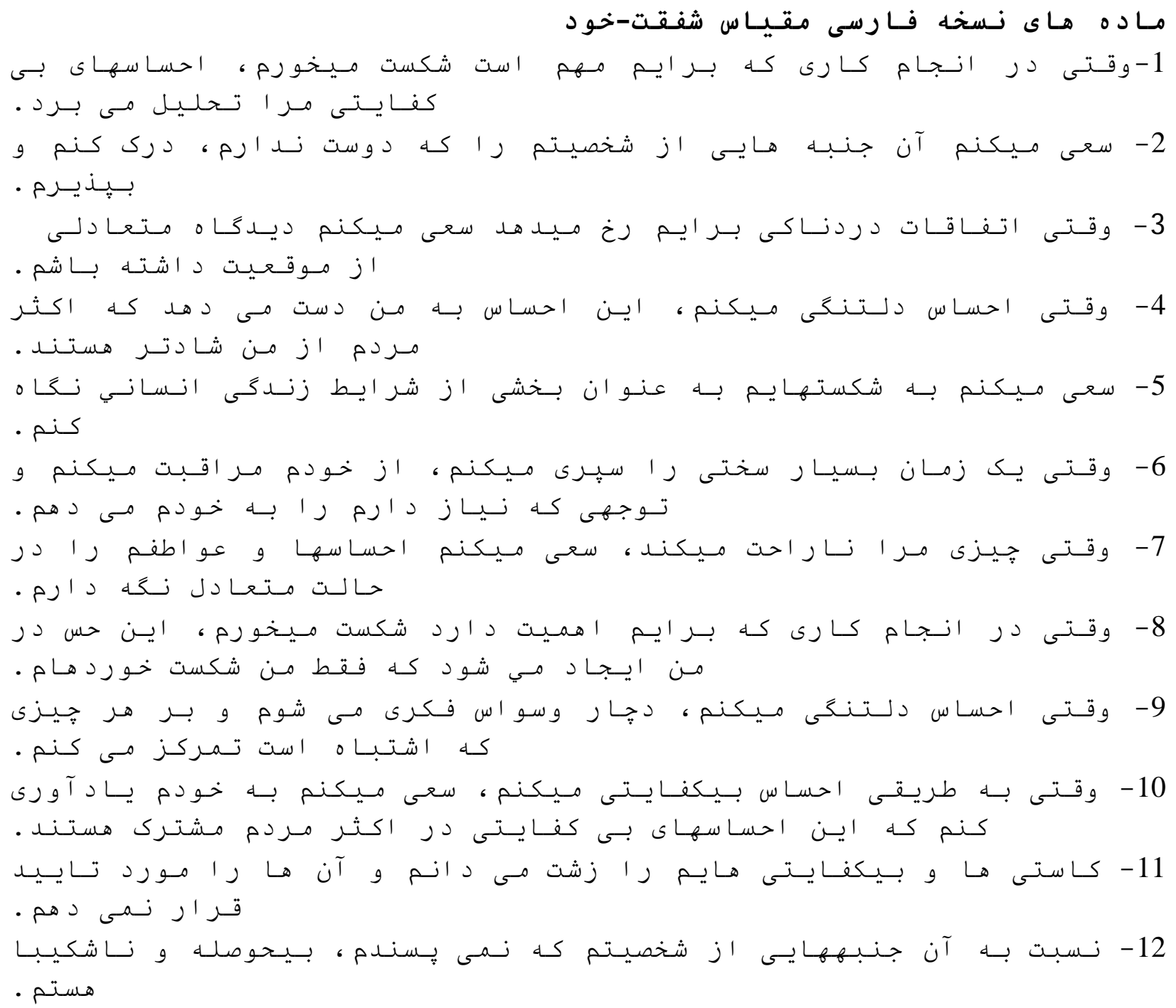


The Reliability and Validity of the Persian Version of Self-Compassion Scale-Revised Edition

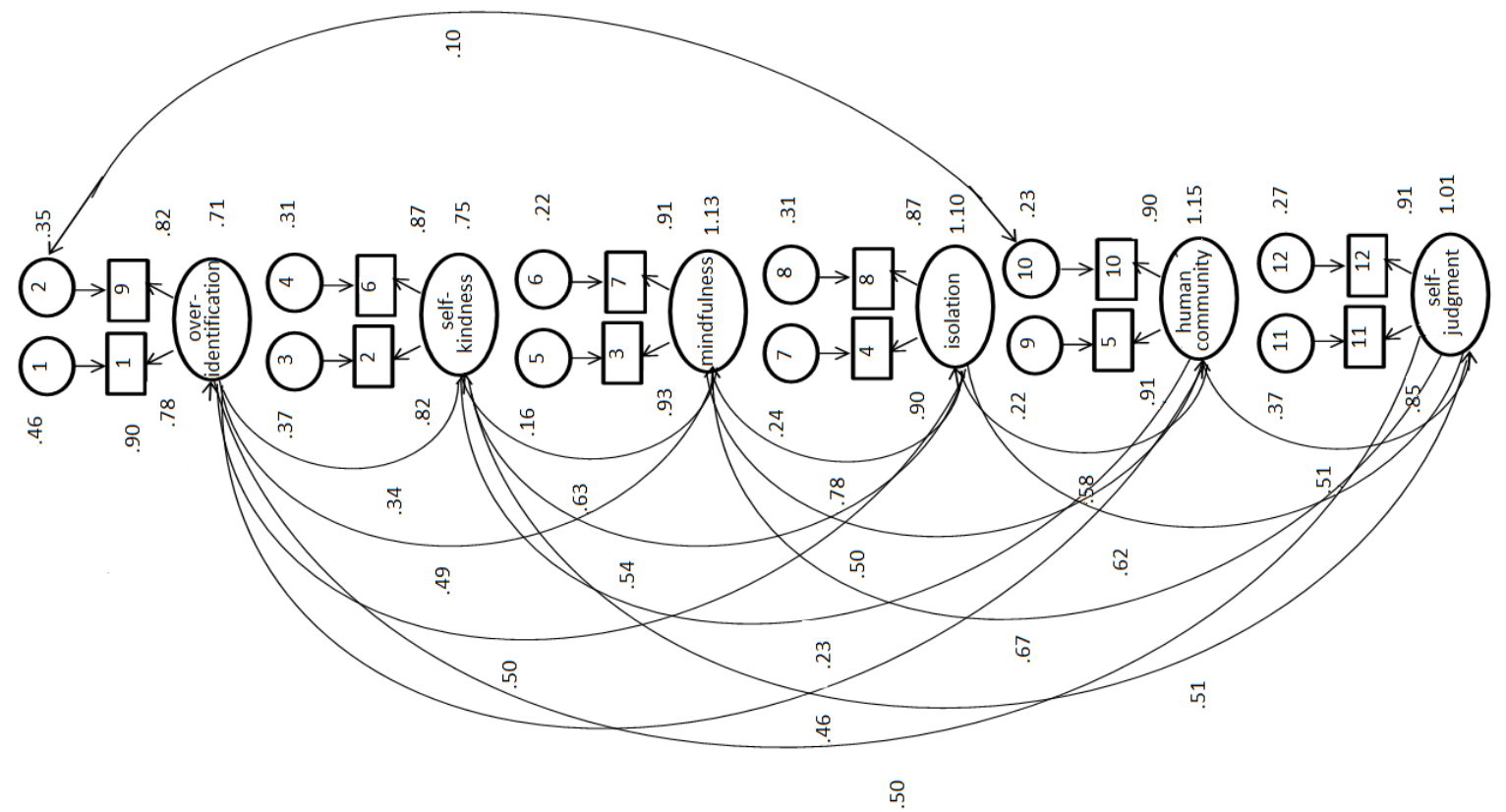

Figure 1. The 6-factor model of the SCS

How to cite this article: A Jelodari, S Gheydari (2016), The Reliability and Validity of the Persian Version of Self-Compassion Scale-Revised Edition, International Journal of Indian Psychology, Volume 3, Issue 3, No. 10, DIP: 18.01.186/20160303, ISBN: 978-1-365-19879-3

(c) The International Journal of Indian Psychology, ISSN 2348-5396 (e)| ISSN: 2349-3429 (p) | 133 